\title{
Evidence of spontaneous spin polarization in the two-dimensional electron gas
}

\author{
A. R. Goñi* ${ }^{1}$ P. Giudici, ${ }^{1}$ F. A. Reboredo, ${ }^{2,3}$ C. R. Proetto,${ }^{3}$ C. Thomsen,${ }^{1}$ K. Eberl,${ }^{4}$ and M. Hauser ${ }^{4}$ \\ ${ }^{1}$ Institut für Festkörperphysik, Technische Universität Berlin, Hardenbergstr. 36, 10623 Berlin, Germany \\ ${ }^{2}$ Lawrence Livermore National Laboratory, Livermore, CA 94551, USA \\ ${ }^{3}$ Comisión Nacional de Energía Atómica, Centro Atómico Bariloche, 8400 S.C. de Bariloche, Argentina \\ ${ }^{4}$ MPI für Festkörperforschung, Heisenbergstr. 1, 70569 Stuttgart, Germany
}

\begin{abstract}
Density-functional calculations using an exact exchange potential for a two-dimensional electron gas (2DEG) formed in a GaAs single quantum well predict the existence of a spin-polarized phase, when an excited subband becomes slightly populated. Direct experimental evidence is obtained from low temperature and low excitation-power photoluminescence (PL) spectra which display the sudden appearance of a sharp emission peak below the energy of the optical transition from the first excited electron subband upon its occupation. The behavior of this PL feature in magnetic fields applied in-plane as well as perpendicular to the 2DEG indicate the formation of spin-polarized domains in the excited subband with in-plane magnetization. For it speaks also the strong enhancement of exchange-vertex corrections observed in inelastic light scattering spectra by spin-density excitations of a slightly occupied first-excited subband.
\end{abstract}

The existence of a spin-polarized phase of the homogeneous electron gas at densities between $10^{20}$ and $10^{18}$ $\mathrm{cm}^{-3}$ and at zero magnetic field, although predicted years ago [1] and later well established from quantum Monte-Carlo calculations [2], remains experimentally unconfirmed. It has been suggested that a partially polarized fluid state [3] might be at the origin of the hightemperature weak ferromagnetism observed recently in La-doped calcium hexaboride [4]. In this case, however, the itinerant magnetism is still a matter of debate, for there might be an alternative explanation in terms of point defects [5]. Modulation-doped semiconductor heterostructures offer a unique opportunity to study the many-body behavior of dilute electron systems due to the extremely long mean-free paths that can be achieved with current growth techniques. Experiments carried out so far, using high-mobility electron gases formed in GaAs/AlGaAs samples, exhibit, upon depletion, the metal-insulator transition at about $2 \times 10^{10} \mathrm{~cm}^{-2}[6,7,8]$ due to the poor screening of the disorder potential of the ionized remote donors. In two dimensions and without many-body screening, this transition should take place for any degree of disorder [9]. Localization effects thus hamper further search for low-density phases of the homogeneous two-dimensional electron gas (2DEG). Here we followed a different approach by raising the Fermi level in a single quantum well (SQW) structure in order to obtain a slightly populated excited subband. By means of photoluminescence (PL) spectroscopy and inelastic light scattering we were able to assess the properties of the dilute component corresponding to the second subband but having the 2DEG always in a metallic state because of the charge present in the ground-state subband.

This Letter reports on possible ferromagnetic order in

${ }^{*}$ ICREA Research Professor, Barcelona, Spain a modulation-doped GaAs SQW, as predicted by selfconsistent density-functional calculations using an exact exchange potential for the 2D electron system [10] and as determined from PL and Raman measurements at low temperatures and excitation powers. Exchange terms of the Coulomb interaction lead to a thermodynamical instability of the 2DEG formed in the quantum well, as soon as the Fermi energy becomes resonant with the bottom of the first-excited subband 11]. At this first-order phase transition the electron gas component corresponding to the excited level breaks into spin-polarized domains with different in-plane magnetization, as expected for planar ferromagnets to minimise the stray field. Evidence for parallel spin alignment is obtained from PL measurements of the light polarization at low magnetic fields and from inelastic light scattering by spin-density excitations involving electronic transitions between the first and second excited subbands.

A 2DEG with a mobility of $8 \times 10^{5} \mathrm{~cm}^{2} / \mathrm{Vs}$ and a density of $6 \times 10^{11} \mathrm{~cm}^{-2}$ at $4.2 \mathrm{~K}$ forms in a modulationdoped $\mathrm{GaAs} / \mathrm{Al}_{0.33} \mathrm{Ga}_{0.67} \mathrm{As}$ quantum well grown by molecular-beam epitaxy. We present results of two samples with 25 and $30 \mathrm{~nm}$ well thickness. The 2DEG is contacted from the surface by In alloying in order to apply a dc bias between it and a metallic back contact. For the 25-nm-wide well and without bias only the lowest subband is occupied with Fermi energy $E_{F} \approx 25 \mathrm{meV}$. The energy separation to the second subband is $E_{01} \approx 28$ $\mathrm{meV}$. For the wider well sample there is a small occupation of the first-excited level at zero voltage. Photoluminescence spectra were excited using low power densities in the range of 0.5 to $5 \mathrm{~W} / \mathrm{cm}^{2}$ and recorded with optical multichannel detection. Magneto-PL measurements were carried out in Faraday as well as Voigt geometry by placing the sample into the cold bore of a $5 \mathrm{~T}$ split-coil magnet. Inelastic light scattering spectra were recorded in backscattering geometry using a line focus in order to avoid heating of the 2DEG at the laser powers required. 
Band-structure calculations of the dilute 2DEG phases were performed in the framework of density-functional theory (DFT) 12. Implementation of DFT requires nontrivial approximations in the exchange and correlation contributions to the total energy. In the widely used local-density approximation (LDA), both exchange and correlation potentials correspond to results obtained for the homogeneous 3D electron gas. A key ingredient in our calculations is the exact treatment of the exchange energy [10], whereas the remaining (small) correlation contribution to the total energy is calculated within LDA. We also approximately accounted for low-temperature effects in the exchange potential. Results obtained by solving the self-consistent Kohn-Sham equations corresponding to the experimental sample geometry are summarized in Fig. 1. For a given electric field which mimics the applied bias, we calculate the total density $n$ and a set of energy eigenvalues $E_{i, \alpha}^{\sigma}$ and eigenfunctions $\xi_{i, \alpha}^{\sigma}$, where $i=0,1$ is the subband index, $\alpha=\mathrm{PI}, \mathrm{PII}, \mathrm{F}$ the phase index and $\sigma=\uparrow, \downarrow$ the spin component. Depending on total density there are three possible configurations: a) The paramagnetic phase denoted PI, where only the ground subband is occupied with equal population for spin-up and down components. This configuration is stable until $E_{F}^{P I}=E_{01, P I}=E_{1, P I}-E_{0, P I}$. b) Phase PII, with both the first and second subbands paramagnetically populated. This configuration is a self-consistent solution at high densities, where $E_{F}^{P I I}=E_{01, P I I} / 2+\pi \hbar^{2} n / 2 m^{*}$, where $m^{*}$ is the electron effective mass. c) A ferromagnetic phase $\mathrm{F}$ for which the lowest subband remains paramagnetic but only one spin component of the second subband (say $\uparrow$ ) is occupied and the other empty. This solution exists for $E_{F}^{F} \geq E_{01, F}^{\uparrow}$ but collapses towards the PII configuration when $E_{F}^{F}=E_{01, F}^{\downarrow}$ because of the kinetic energy cost to maintain a single spin population in the second subband.

Figure 1 shows two remarkable effects, both beyond the reach of LDA: The abrupt decrease of the intersubband spacing $E_{01}$ at about $0.72 \times 10^{12} \mathrm{~cm}^{-2}$ when transforming from phase PI to PII and the very existence of the spinpolarized phase $\mathrm{F}$. The sudden renormalization of $E_{01}$, which is a consequence of intersubband exchange terms, has been discussed in Ref. [11]. For an incipient population of the excited subband the leading exchange contribution to the 2DEG compressibility is negative, such that the electron system becomes thermodynamically unstable against the shift of a macroscopic amount of charge from the ground to the first-excited subband. When $E_{F}=E_{01}$ electrons start to be transferred from states at the Fermi surface of the lowest subband to Brillouinzone center states in the excited 2D level. This leads to the discontinuities observed in PL associated with the spontaneous breaking of translational invariance.

In contrast, spin polarization is favored by intrasubband exchange terms at low densities of the excited sub-

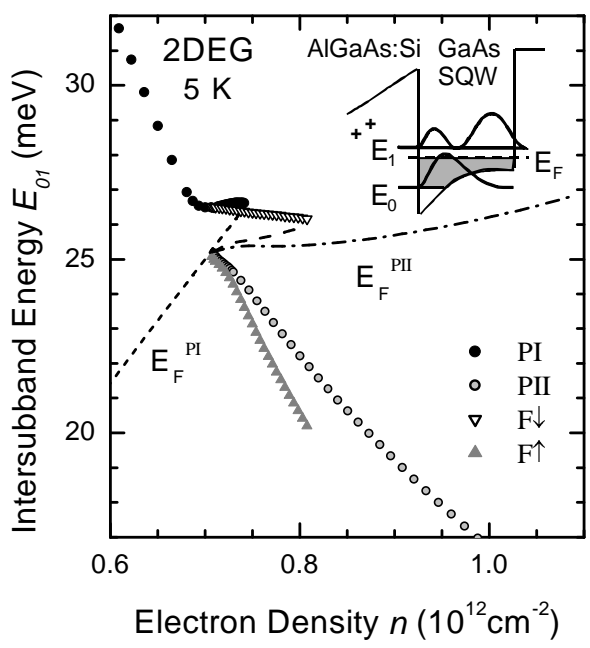

FIG. 1: Dependence on total density $n$ of the intersubband energy $E_{01}$ calculated within exact-exchange theory for a 25nm-wide SQW at $5 \mathrm{~K}$. Different symbols correspond to the paramagnetic solutions with one (PI) and two (PII) occupied subbands and the ferromagnetic phase $(\mathrm{F})$, as indicated. Lines indicate the position of the Fermi level in each phase. The inset shows a sketch of the conduction band profile of the SQW.

band $n_{1}=n_{1 \downarrow}+n_{1 \uparrow}$, yielding a negative non-linear contribution that goes as $-n_{1 \sigma}^{3 / 2}$. With $n_{1 \uparrow, \downarrow}=\left(\frac{n_{1}}{2} \mp \delta n_{1}\right)$ it is given by

$$
n_{1 \uparrow}^{3 / 2}+n_{1 \downarrow}^{3 / 2} \approx 2\left(\frac{n_{1}}{2}\right)^{3 / 2} \cdot\left[1+\frac{3}{2}\left(\frac{\delta n_{1}}{n_{1}}\right)^{2}\right] .
$$

Thus, the system gains energy by unbalancing the spin population of the second subband. The last term in Eq. (1) is an exchange-hole energy gain in the excited subband, which reduces the overall Coulomb repulsion in the 2DEG due to the Pauli principle. With increasing $n_{1}$, positive contributions from the kinetic and Hartree energies overcome the intrasubband exchange part and the F phase collapses towards the paramagnetic PII phase.

Direct evidence of the existence of the F phase is obtained from PL spectra recorded at low temperatures using very low laser excitation. Figure 2 shows a set of spectra of the 25 -nm-wide SQW taken at $1.7 \mathrm{~K}$ and $3.5 \mu \mathrm{W}$ laser power with different gate voltage. As the Fermi level is raised towards resonance with the second subband, an additional peak becomes apparent at about $1.2 \mathrm{meV}$ below the energy $E_{1}$ of optical recombination processes from the first excited electron subband. The $\mathrm{F}$ feature literally pops up in PL, increasing in intensity while merging together with the $E_{1}$ peak before its abrupt renormalization takes place. We emphasize that peak $\mathrm{F}$ is only observable if the lattice temperature is kept below $5 \mathrm{~K}$ and/or the power density does not exceed $10 \mathrm{~W} / \mathrm{cm}^{2}$. On the contrary, at higher excitation 


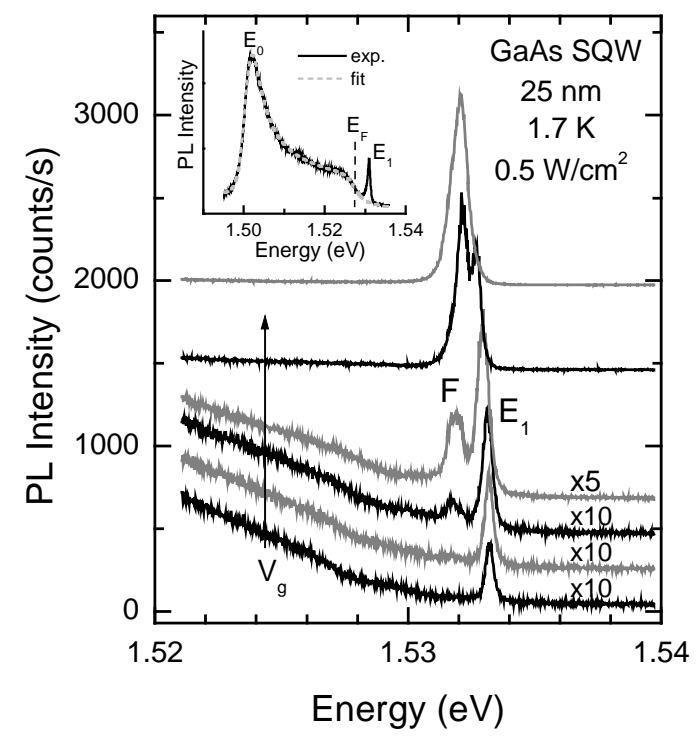

FIG. 2: PL spectra of the 25-nm-wide SQW in the energy region of the $E_{1}$ transition for different gate bias $\mathrm{V}_{g}$ taken at $1.7 \mathrm{~K}$ with $0.5 \mathrm{~W} / \mathrm{cm}^{2}$ power density. As an example, the inset displays a full-range PL spectrum (solid curve) and the fitting function (dashed line) used for lineshape analysis 7].

levels the $E_{1}$ peak instead of disappearing displays just a slight line broadening and a strong increase in intensity. We also note that close to the PI-PII phase transition the 2DEG is very unstable, a situation which is reflected in the uncontrolled change of the Fermi level, and thus of the PL spectrum, even without varying the external voltage. In fact, the spectra of Fig. 2 correspond to snapshots of $1 \mathrm{~s}$ during cycles where the electron system goes back and forth having successively different occupations of the second subband. The first order of the transition manifests itself in the transformation of the $E_{1}$ lineshape from Lorentzian to Gaussian and back to Lorentzian during the process of renormalization. Such a change from homogeneous (lifetime) line broadening to an inhomogeneous one (level distribution) is consistent with the formation of density domains in the second subband during the phase transition. The $\mathrm{F}$ feature is then associated with the recombination of electrons within the domains, whereas the peak $E_{1}$ arises from hot luminescence coming from regions of the SQW plane where the second subband is still empty.

The energy position of the $E_{1}$ and $\mathrm{F}$ peaks measured at $2 \mathrm{~K}$ with $20 \mu \mathrm{W}$ is plotted in Fig. 3 as a function of the Fermi energy. When the Fermi energy equals $E_{1}$, an abrupt subband renormalization by about $2 \mathrm{meV}$ occurs. Afterwards, one finds the 2DEG in the paramagnetic phase PII and the variation of the subband energy with bias, i.e. Fermi level, is continuous but exhibits a clear hysteresis. The stability range of the $\mathrm{F}$ phase comprises Fermi energies only within $3 \mathrm{meV}$ below $E_{1}$, and

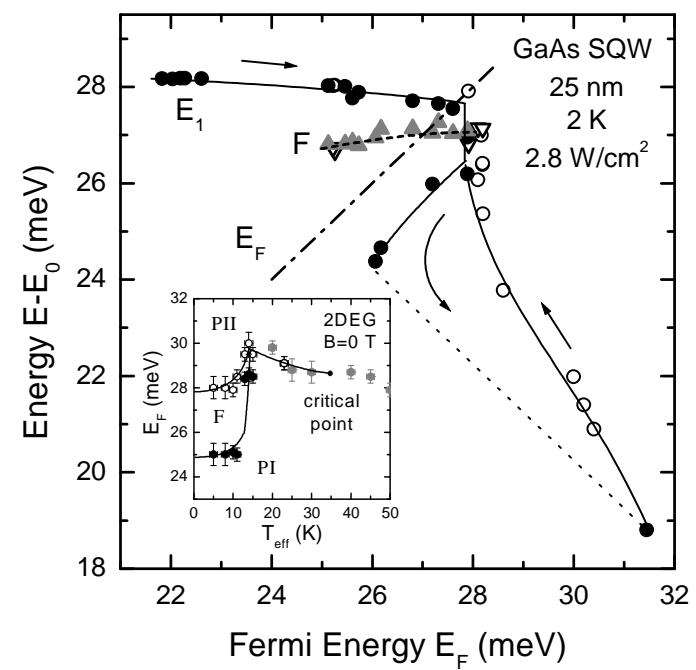

FIG. 3: Energy position of the $E_{1}$ and $\mathrm{F}$ peaks referred to $E_{0}$, the bottom of the ground subband, versus Fermi energy. The corresponding electron temperature is $10 \mathrm{~K}$. Solid (open) symbols correspond to data taken with increasing (decreasing) bias. Solid lines are a guide to the eye. The inset shows the phase diagram of the 2DEG at zero magnetic field obtained from PL measurements. Grey data points are results of Ref. 11] obtained at high power densities.

in this region the energy of the $\mathrm{F}$ peak is almost independent of the Fermi level. This is a very important point, for it rules out an explanation of our observations in terms of the Fermi-edge singularity [13].

The inset to Fig. 3 illustrates the phase diagram of the 2DEG conceived from the sets of data obtained at different temperatures and laser powers. Effective electron temperatures were determined from the width of the Fermi edge in PL spectra. Below a sample temperature of $25 \mathrm{~K}$, the temperature of the 2DEG is mainly given by the excitation-power level. The lowest value of $5 \mathrm{~K}$ is obtained with the sample immersed in superfluid helium and using only around $1 \mu \mathrm{W}$ of laser power. The region in the phase diagram representing the stability range of the F phase corresponds to the Fermi levels and temperatures for which the peak $\mathrm{F}$ is apparent in PL. At $13 \mathrm{~K}$, where the $\mathrm{F}$ phase collapses into the PII one, there is a triple point. We note that this temperature corresponds to the formation energy of the $\mathrm{F}$ phase, as given by the difference between $E_{1}$ and $\mathrm{F}$ peak energy. As reported earlier 11], there is a critical point at $\mathrm{T}_{C}=35 \mathrm{~K}$. For completeness we point out that the same phenomenology has been observed in three other SQW samples with different well widths.

Having experimentally proven the existence of an additional phase of the 2DEG, we now present the evidence for ferromagnetic order stemming from magneto-PL measurements. We consider first the case of magnetic fields applied perpendicular to the plane of the SQW (Fara- 
day geometry). Whereas the $E_{1}$ peak splits into two Zeeman components with clearly different circular polarization $\sigma^{+}, \sigma^{-}$with the expected gyromagnetic factor 14], the $\mathrm{F}$ peak does not show any splitting at all nor any degree of circular polarization. On the contrary, its intensity decreases linearly with field, disappearing at about $1.5 \mathrm{~T}$. Such behavior is compatible with the assumption that the magnetization within the density domains is randomly, but in-plane, oriented. A magnetic field in the perpendicular direction destroys the domains since $\mathrm{B}_{\perp}$ significantly reduces the wave-function overlap in the second subband and thus the intrasubband exchange interaction.

For magnetic fields applied along the 2DEG plane the intensity of the $\mathrm{F}$ peak remains essentially constant. In contrast, its degree of linear polarization defined as $\gamma=$ $\left(I_{V}-I_{H}\right) /\left(I_{V}+I_{H}\right)$ increases linearly with $\mathrm{B}_{\|}$by $30 \%$ in the range from zero to $0.6 \mathrm{~T}$. Here $I_{V}$ and $I_{H}$ are the intensities of the $\mathrm{F}$ peak for vertical and horizontal linear polarization, respectively, normalized to the intensity of the PL spectrum in the energy region just underneath the Fermi edge, where the emission is expected to be independent of its polarization. The field is aligned along the horizontal direction, but rotated $90^{\circ}$ with respect to the direction of light collection (Voigt configuration). In this geometry, light emitted with circular polarization along the field is detected as being vertically polarized. Hence, the observed linear increase in $\gamma$ can be ascribed to the successive orientation of the domain magnetization parallel to the external field. Thus, $\gamma$ is given by the Brillouin function $B_{J}$ which in the low-field limit yields [15] $\gamma=(J+1) g \mu_{B} B_{\|} /\left(3 k_{B} T_{\text {eff }}\right)$. Here is $J$ the average total spin of the domains in units of $\hbar / 2, g=-0.44$ is the gyromagnetic factor of GaAs [14], $\mu_{B}$ is the Bohr magneton, $k_{B}$ is the Boltzmann constant, and $T_{\text {eff }}$ is the electron temperature. From the measured slope for $\gamma$ we infer an average number of $1 / 2^{2}$-spins, i.e. electrons, within a domain of about 50 (25 nm SQW). For a typical electron density of $5 \times 10^{10} \mathrm{~cm}^{-2}$ corresponding to the jump at the PI-PII phase transition the mean radius of a domain should be around $200 \mathrm{~nm}$.

Another striking result which speaks for a spin polarization of the dilute electron system in subband ' 1 ' is obtained from inelastic light scattering. Collective spin-density (SD) excitations of the 2DEG are known to be shifted down in energy from the intersubband singleparticle (SP) gap by many-body effects, the so-called excitonic shift. It represents the vertex correction due to exchange-correlation terms of the Coulomb interaction [16. The determination of its magnitude is straightforward from the energies of the excitations $\hbar \omega_{S P}$ and $\hbar \omega_{S D}$ measured in depolarized light scattering spectra, according to [16]: $-2 \gamma_{S D} n_{2 D}=\frac{\omega_{S D}^{2}-\omega_{S P}^{2}}{\omega_{S P}}$. Here $\gamma_{S D}$ represents the exchange-correlation energy and $n_{2 D}$ is the electron density of the occupied subband. Figure 4 shows the val-

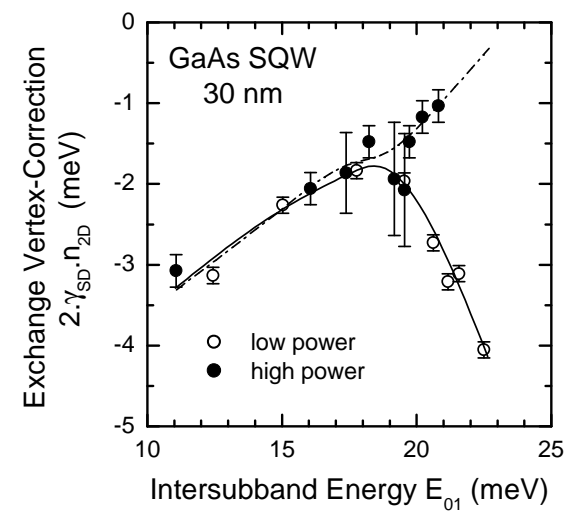

FIG. 4: Exchange-vertex correction to the spin-density excitation involving electronic transitions between subbands '1' and '2' as a function of the intersubband spacing between the two lowest subbands of a 30-nm-wide quantum well. Open(closed) symbols correspond to measurements using low(high) power densities, i.e. the effective electron temperature being 5 and $15 \mathrm{~K}$, respectively. Curves are a guide to the eye.

ues of the exchange vertex correction to the spin-density excitation associated with electronic transitions between the first and second excited subbands of a 30-nm-wide SQW. In the graph, the first-excited subband is being filled for decreasing subband spacing $E_{01}$. Open symbols correspond to data obtained at a very low power density such that the F peak is apparent in PL. For comparison the full circles represent the high-power case, where the effective electron temperature is too high for the spinpolarized phase to exist (peak $\mathrm{F}$ is no longer observable). The exchange vertex correction exhibits a strong enhancement at low electron temperatures and very low filling of subband '1',i.e. in the stability range of phase $\mathrm{F}$, whereas at high power levels it extrapolates to almost zero. The results are independent of laser power, when the renormalization of $E_{1}$ sets in. As demonstrated earlier for fractional quantum Hall states with odd filling factor 17] and in GaAs quantum wires in magnetic fields 18], this is clear indication of spin polarization. The cost in exchange energy to flip a spin in a ferromagnetically ordered state exceeds many times that of the nonmagnetic case. We emphasize that such an enhancement of vertex corrections is clearly observed for the 1-2 electronic transitions rather than the ' 0,1 ' subbands, for in the latter case most of the electrons involved in the spin excitation are in a paramagnetic state.

We thank F. Guinea for inspiring discussions and W. Dietsche for assistance with the sample design and growth. F.A.R. acknowledges the auspices of the U.S. Dept. of Energy at the University of California/Lawrence Livermore National Lab under contract no. W-7405-Eng48. P.G. thanks the DAAD for financial support. This work is supported in part by the DFG in the framework of Sfb 296. 
[1] F. Bloch, Z. Phys. 57, 545 (1929).

[2] D.M. Ceperley and B.J. Alder, Phys. Rev. Lett. 45, 566 (1980); F. Rapisarda and G. Senatore, Austr. J. Phys. 49, 161 (1996); C. Attaccalite, S. Moroni, P. Gori-Giorgi, and G.B. Bachelet, Phys. Rev. Lett. 88, 256601 (2002).

[3] D.M. Ceperley, Nature 397, 386 (1999).

[4] D.P. Young, D. Hall, M.D. Torelli, Z. Fisk, J.L. Sarrao, J.D. Thompson, H.-R. Ott, S.B. Oseroff, R.G. Goodrich, and R. Zysler, Nature 397, 412 (1999).

[5] R. Monnier and B. Delley, Phys. Rev. Lett. 87, 157204 (2001); I.S. Elfimov, S. Yunoki, and G.A. Sawatzky, Phys. Rev. Lett. 89, 216403 (2002).

[6] S.V. Kravchenko, G.V. Kravchenko, J.E. Furneaux, V.M. Pudalov, and M. D'Iorio, Phys. Rev. B 50, 8039 (1994); Y. Hanein, U. Meirav, D. Shahar, C.C. Li, D.C. Tsui, and H. Shtrikman, Phys. Rev. Lett. 80, 1288 (1998).

[7] S. Ernst, A.R. Goñi, K. Syassen, and K. Eberl, Phys. Rev. Lett. 72, 4029 (1994).

[8] S. Ilani, A. Yacoby, D. Mahalu, and H. Shtrikman, Sci. 292, 1354 (2001).

[9] E. Abrahams, P.W. Anderson, D.C. Licciardello, and T.V. Ramakrishnan, Phys. Rev. Lett. 42, 673 (1979).

[10] F.A. Reboredo and C.R. Proetto, Phys. Rev. B 67,
115325 (2003); S. Rigamonti, F.A. Reboredo, and C.R. Proetto, Phys. Rev. B 68, 235309 (2003).

[11] A.R. Goñi, U. Haboeck, C. Thomsen, K. Eberl, F.A. Reboredo, C.R. Proetto, and F. Guinea, Phys. Rev. B 65, R121313 (2002).

[12] W. Kohn, Rev. Mod. Phys. 71, 1253 (1999).

[13] W. Chen, M. Fritze, W. Walecki, A.V. Nurmikko, D. Ackley, J.M. Hong, and L.L. Chang, Phys. Rev. B 45, 8464 (1992).

[14] M.J. Snelling, E. Blackwood, C.J. McDonagh, R.T. Harley, and C.T.B. Foxon, Phys. Rev. B 45, R3922 (1992).

[15] D. Craik, in Magnetism. Principles and applications (Wiley, Chichester, 1995).

[16] A. Pinczuk, S. Schmitt-Rink, G. Danan, J.P. Valladares, L.N. Pfeiffer, and K.W. West, Phys. Rev. Lett. 63, 1633 (1989); D. Gammon, B.V. Shanabrook, J.C. Ryan, and D.S. Katzer, Phys. Rev. Lett. 68, 1884 (1992).

[17] A. Pinczuk, B.S. Dennis, D. Heiman, C. Kallin, L. Brey, C. Tejedor, S. Schmitt-Rink, L.N. Pfeiffer, and K.W. West, Phys. Rev. Lett. 68, 3623 (1992).

[18] A.R. Goñi, A. Pinczuk, J.S. Weiner, B.S. Dennis, L.N. Pfeiffer, and K.W. West, Phys. Rev. Lett. 70, 1151 (1993). 